\title{
Existence of solutions for integro-differential equations of fractional order with nonlocal three-point fractional boundary conditions
}

\author{
Ravi P Agarwal ${ }^{1,2}$, Sotiris K Ntouyas ${ }^{3}$, Bashir Ahmad ${ }^{2 *}$ and Mohammed S Alhothuali ${ }^{2}$
}

\section{"Correspondence: \\ bashir_qau@yahoo.com \\ ${ }^{2}$ Department of Mathematics, \\ Faculty of Science, King Abdulaziz \\ University, P.O. Box 80203, Jeddah, \\ 21589, Saudi Arabia \\ Full list of author information is \\ available at the end of the article}

\begin{abstract}
In this paper, we study the existence of solutions for Riemann-Liouville type integro-differential equations of fractional order $\alpha \in(2,3]$ with nonlocal three-point fractional boundary conditions via Sadovskii's fixed point theorem for condensing maps. An illustrative example is also presented.

MSC: 34A08; 34B10; 34B15

Keywords: fractional differential equations; nonlocal integral boundary conditions; existence; fixed point theorems
\end{abstract}

\section{Introduction}

Fractional calculus has recently evolved as an interesting and important field of research. The much interest in the subject owes to its extensive applications in the mathematical modeling of several phenomena in many engineering and scientific disciplines such as physics, chemistry, biophysics, biology, blood flow problems, control theory, aerodynamics, nonlinear oscillation of earthquake, the fluid-dynamic traffic model, polymer rheology, regular variation in thermodynamics, economics, fitting of experimental data, etc. [1,2]. A significant feature of a fractional order differential operator, in contrast to its counterpart in classical calculus, is its nonlocal behavior. It means that the future state of a dynamical system or process based on the fractional differential operator depends on its current state as well its past states. It is equivalent to saying that differential equations of arbitrary order are capable of describing memory and hereditary properties of certain important materials and processes. This aspect of fractional calculus has contributed towards the growing popularity of the subject.

Nonlocal initial and boundary value problems of nonlinear fractional order differential equations have recently been investigated by several researchers. The domain of study ranges from the theoretical aspects (like existence, uniqueness, periodicity, asymptotic behavior, etc.) to the analytic and numerical methods for fractional differential equations. In fact the theory of differential equations of fractional order (parallel to the well-known theory of ordinary differential equations) has been growing independently for the last three decades. For some recent development of the subject, we refer, for instance, to a series of papers [3-14] and references cited therein.

In this paper, we discuss the existence of solutions for a boundary value problem of integro-differential equations of fractional order with nonlocal three-point fractional

(c) 2013 Agarwal et al: licensee Springer. This is an Open Access article distributed under the terms of the Creative Commons Attribution License (http://creativecommons.org/licenses/by/2.0), which permits unrestricted use, distribution, and reproduction in any medium, provided the original work is properly cited. 
boundary conditions

$$
\left\{\begin{array}{l}
-D^{\alpha} x(t)=A f(t, x(t))+B I^{\beta} g(t, x(t)), \quad 2<\alpha \leq 3, t \in[0,1], \\
D^{\delta} x(0)=0, \quad D^{\delta+1} x(0)=0, \quad D^{\delta} x(1)-D^{\delta} x(\eta)=a,
\end{array}\right.
$$

where $0<\delta \leq 1, \alpha-\delta>3,0<\beta<1,0<\eta<1, D^{(\cdot)}$ denotes the Riemann-Liouville fractional derivative of order $(\cdot), f, g$ are given continuous functions, and $A, B, a$ are real constants.

The objective of the present work is to establish the existence of solutions for the given problem by applying Sadovskii's fixed point theorem for condensing maps. It is imperative to note that the application of Sadovskii's fixed point theorem for condensing maps in the present scenario is new. Moreover, the right-hand side of the fractional differential equation in (1) provides a liberty to fix it in terms of non-integral and integral terms. Observe that the integral term in (1) is a Riemann-Liouville integral of order $\beta \in(0,1)$, which reduces to the classical integral term $\left(\int_{0}^{t} g(s, x(s)) d s\right)$ in the limit $\beta \rightarrow 1^{-}$. The nature of the nonlinearity considered in the problem (1) becomes of non-integral type if we take $B=0$ in (1) and corresponds to integral type for $A=0$ in (1). Furthermore, the given boundary conditions are also interesting and important from a physical point of view $[15,16]$ as the condition $D^{\delta} x(1)-D^{\delta} x(\eta)=a$ is a fractional analogue of the classical flux condition $x^{\prime}(1)-x^{\prime}(\eta)=a$ (the difference of flux values at the right end point and at an intermediate point of the interval $[0,1]$ remains constant).

\section{Auxiliary results}

We recall here the following definitions.

Definition 2.1 The Riemann-Liouville fractional integral of order $q$ for a continuous function $g$ is defined as

$$
I^{q} g(t)=\frac{1}{\Gamma(q)} \int_{0}^{t} \frac{g(s)}{(t-s)^{1-q}} d s, \quad q>0
$$

provided the integral exists.

Definition 2.2 The Riemann-Liouville derivative of fractional order $q$ for a continuous function $g:(0, \infty) \rightarrow \mathbb{R}$ is defined as

$$
D_{0+}^{q} g(t)=\frac{1}{\Gamma(n-q)}\left(\frac{d}{d t}\right)^{n} \int_{0}^{t}(t-s)^{n-q-1} g(s) d s, \quad n=[q]+1,
$$

where $[q]$ denotes the integer part of the real number $q$.

By the substitution $x(t)=I^{\delta} y(t)=D^{-\delta} y(t)$, where $y(t)$ is a suitable continuous function, the problem (1) takes the form

$$
\left\{\begin{array}{l}
-D^{\alpha-\delta} y(t)=A f\left(t, I^{\delta} y(t)\right)+B I^{\beta} g\left(t, I^{\delta} y(t)\right), \quad t \in[0,1], \\
y(0)=0, \quad y^{\prime}(0)=0, \quad y(1)-y(\eta)=a .
\end{array}\right.
$$


Lemma 2.1 For any $h \in C(0,1) \cap L(0,1)$, the unique solution of the linear fractional boundary value problem

$$
\left\{\begin{array}{l}
-D^{\alpha-\delta} y(t)=h(t), \quad t \in[0,1], \\
y(0)=0, \quad y^{\prime}(0)=0, \quad y(1)-y(\eta)=a,
\end{array}\right.
$$

is

$$
y(t)=-I^{\alpha-\delta} h(t)+\frac{t^{\alpha-\delta-1}}{1-\eta^{\alpha-\delta-1}}\left(a+I^{\alpha-\delta} h(1)-I^{\alpha-\delta} h(\eta)\right) .
$$

Proof It is well known that the solutions of the fractional differential equation in (3) can be written as [2]

$$
y(t)=-I^{\alpha-\delta} h(t)+c_{1} t^{\alpha-\delta-1}+c_{2} t^{\alpha-\delta-2}+c_{3} t^{\alpha-\delta-3},
$$

where $c_{1}, c_{2}, c_{3} \in \mathbb{R}$ are arbitrary constants. Using the given boundary conditions, we find that $c_{2}=0, c_{3}=0$ and

$$
c_{1}=\frac{1}{1-\eta^{\alpha-\delta-1}}\left(a+I^{\alpha-\delta} h(1)-I^{\alpha-\delta} h(\eta)\right) .
$$

Substituting these values in (4), we get

$$
y(t)=-I^{\alpha-\delta} h(t)+\frac{t^{\alpha-\delta-1}}{1-\eta^{\alpha-\delta-1}}\left(a+I^{\alpha-\delta} h(1)-I^{\alpha-\delta} h(\eta)\right) .
$$

This completes the proof.

Thus, the solution of a linear variant of the problem (1) can be written as

$$
\begin{aligned}
x(t) & =I^{\delta} y(t) \\
& =I^{\delta}\left[-I^{\alpha-\delta} h(t)+\frac{t^{\alpha-\delta-1}}{1-\eta^{\alpha-\delta-1}}\left(a+I^{\alpha-\delta} h(1)-I^{\alpha-\delta} h(\eta)\right)\right] \\
& =-I^{\alpha} h(t)+\frac{1}{1-\eta^{\alpha-\delta-1}}\left(a+I^{\alpha-\delta} h(1)-I^{\alpha-\delta} h(\eta)\right) \int_{0}^{t} \frac{(t-s)^{\delta-1}}{\Gamma(\delta)} s^{\alpha-\delta-1} d s \\
& =-I^{\alpha} h(t)+\frac{1}{1-\eta^{\alpha-\delta-1}}\left(a+I^{\alpha-\delta} h(1)-I^{\alpha-\delta} h(\eta)\right)\left\{\frac{t^{\alpha-1}}{\Gamma(\delta)} \int_{0}^{1}(1-v)^{\delta-1} v^{\alpha-\delta-1} d v\right\},
\end{aligned}
$$

where we have used the substitution $s=v t$ in the integral of the last term. Using the relation for beta function $B(\cdot, \cdot)$,

$$
B(\beta+1, \alpha)=\int_{0}^{1}(1-u)^{\alpha-1} u^{\beta} d u=\frac{\Gamma(\alpha) \Gamma(\beta+1)}{\Gamma(\alpha+\beta+1)},
$$

we get

$$
x(t)=-I^{\alpha} h(t)+\frac{\Gamma(\alpha-\delta) t^{\alpha-1}}{\left(1-\eta^{\alpha-\delta-1}\right) \Gamma(\alpha)}\left(a+I^{\alpha-\delta} h(1)-I^{\alpha-\delta} h(\eta)\right) .
$$


The solution of the original nonlinear problem (1) can be obtained by replacing $h$ with the right-hand side of the fractional equation of (1) in (5).

Let $\mathcal{C}=C([0,1], \mathbb{R})$ denote the Banach space of all continuous functions from $[0,1] \rightarrow \mathbb{R}$ endowed with the norm defined by $\|x\|=\sup \{|x(t)|, t \in[0,1]\}$.

Definition 2.3 Let $M$ be a bounded set in a metric space $(X, d)$, then the Kuratowskii measure of noncompactness $\alpha(M)$ is defined as inf $\{\epsilon: M$ covered by a finitely many sets such that the diameter of each set $\leq \epsilon\}$.

Definition 2.4 [17] Let $\Phi: D(\Phi) \subseteq X \rightarrow X$ be a bounded and continuous operator on a Banach space $X$. Then $\Phi$ is called a condensing map if $\alpha(\Phi(B))<\alpha(B)$ for all bounded sets $B \subset D(\Phi)$, where $\alpha$ denotes the Kuratowski measure of noncompactness.

Lemma 2.2 [18, Example 11.7] The map $K+C$ is a $k$-set contraction with $0 \leq k<1$, and thus also condensing, if

(i) $K, C: D \subseteq X \rightarrow X$ are operators on the Banach space $X$;

(ii) $K$ is $k$-contractive, i.e.,

$$
\|K x-K y\| \leq k\|x-y\|
$$

for all $x, y \in D$ and fixed $k \in[0,1)$;

(iii) $C$ is compact.

Theorem 2.1 [19] Let $B$ be a convex, bounded and closed subset of a Banach space $X$ and let $\Phi: B \rightarrow B$ be a condensing map. Then $\Phi$ has a fixed point.

\section{Main result}

In the following we denote by $L_{1 / p}\left([0,1], \mathbb{R}^{+}\right)$the space of $\frac{1}{p}$-Lebesgue measurable functions from $[0,1]$ to $\mathbb{R}^{+}$with the norm $\|\mu\|=\left(\int_{0}^{1}|\mu(s)|^{\frac{1}{p}} d s\right)^{p}$.

Theorem 3.1 Let $f, g:[0,1] \times \mathbb{R} \rightarrow \mathbb{R}$ be continuous functions satisfying the following conditions:

$\left(\mathrm{H}_{1}\right)$ f satisfies the Lipschitz condition

$$
|f(t, x)-f(t, y)| \leq L_{1}(t)|x-y| \quad \text { for all }(t, x),(t, y) \in[0,1] \times \mathbb{R},
$$

where $L_{1} \in L_{1 / p}\left([0,1], \mathbb{R}^{+}\right), p=\min \left\{q_{1}, q_{2}\right\}, q_{1} \in(0,1), q_{2} \in(0, \alpha-\delta) ;$

$\left(\mathrm{H}_{2}\right)$ there exist a function $m \in L_{1 / p}\left([0,1], \mathbb{R}^{+}\right)$and a nondecreasing function $\psi: \mathbb{R}^{+} \rightarrow \mathbb{R}^{+}$ such that

$$
|g(t, x)| \leq m(t) \psi(\|x\|), \quad \forall(t, x) \in[0,1] \times \mathbb{R} .
$$

Then the boundary value problem (1) has at least one solution, provided

$$
\gamma:=\frac{|A|\left\|L_{1}\right\|}{\Gamma(\alpha)}\left(\frac{1-p}{\alpha-p}\right)^{1-p}+\frac{|A||Q|\left\|L_{1}\right\|}{\Gamma(\alpha-\delta)}\left(\frac{1-p}{\alpha-\delta-p}\right)^{1-p}\left(1+\eta^{\alpha-\delta-p}\right)<1 .
$$


Proof Let $B_{r}=\{x \in \mathcal{C}:\|x\| \leq r\}$ be a closed bounded and convex subset of $\mathcal{C}:=C([0,1], \mathbb{R})$, where $r$ will be fixed later. Using (5), we define a map $\Phi: B_{r} \rightarrow \mathcal{C}$ as

$$
\begin{aligned}
(\Phi x)(t)= & -A \int_{0}^{t} \frac{(t-s)^{\alpha-1}}{\Gamma(\alpha)} f(s, x(s)) d s-B \int_{0}^{t} \frac{(t-s)^{\alpha+\beta-1}}{\Gamma(\alpha+\beta)} g(s, x(s)) d s \\
& +Q t^{\alpha-1}\left[a+A \int_{0}^{1} \frac{(1-s)^{\alpha-\delta-1}}{\Gamma(\alpha-\delta)} f(s, x(s)) d s\right. \\
& +B \int_{0}^{1} \frac{(1-s)^{\alpha-\delta+\beta-1}}{\Gamma(\alpha-\delta+\beta)} g(s, x(s)) d s \\
& \left.-A \int_{0}^{\eta} \frac{(\eta-s)^{\alpha-\delta-1}}{\Gamma(\alpha-\delta)} f(s, x(s)) d s-B \int_{0}^{\eta} \frac{(\eta-s)^{\alpha-\delta+\beta-1}}{\Gamma(\alpha-\delta+\beta)} g(s, x(s)) d s\right],
\end{aligned}
$$

where

$$
Q=\frac{\Gamma(\alpha-\delta)}{\left(1-\eta^{\alpha-\delta-1}\right) \Gamma(\alpha)}, \quad \eta^{\alpha-\delta-1} \neq 1 .
$$

Observe that the problem (1) is equivalent to a fixed point problem $\Phi x=x$.

Let us decompose $\Phi$ as $\Phi=\Phi_{1}+\Phi_{2}$, where

$$
\begin{aligned}
\left(\Phi_{1} x\right)(t)= & -A \int_{0}^{t} \frac{(t-s)^{\alpha-1}}{\Gamma(\alpha)} f(s, x(s)) d s+a Q t^{\alpha-1} \\
& +A Q t^{\alpha-1}\left[\int_{0}^{1} \frac{(1-s)^{\alpha-\delta-1}}{\Gamma(\alpha-\delta)} f(s, x(s)) d s\right. \\
& \left.-\int_{0}^{\eta} \frac{(\eta-s)^{\alpha-\delta-1}}{\Gamma(\alpha-\delta)} f(s, x(s)) d s\right], \quad t \in[0,1]
\end{aligned}
$$

and

$$
\begin{aligned}
\left(\Phi_{2} x\right)(t)= & -B \int_{0}^{t} \frac{(t-s)^{\alpha+\beta-1}}{\Gamma(\alpha+\beta)} g(s, x(s)) d s \\
& +B Q t^{\alpha-1}\left[\int_{0}^{1} \frac{(1-s)^{\alpha-\delta+\beta-1}}{\Gamma(\alpha-\delta+\beta)} g(s, x(s)) d s\right. \\
& \left.-\int_{0}^{\eta} \frac{(\eta-s)^{\alpha-\delta+\beta-1}}{\Gamma(\alpha-\delta+\beta)} g(s, x(s)) d s\right], \quad t \in[0,1] .
\end{aligned}
$$

Step 1. $\Phi\left(B_{r}\right) \subset B_{r}$.

For that, we select $r \geq \frac{\omega}{1-\gamma}$, where

$$
\begin{aligned}
& \omega=|A| \sigma+\frac{|B|\left\|m_{2}\right\| \psi(r)}{\Gamma(\alpha+\beta)}\left(\frac{1-p}{\alpha+\beta-p}\right)^{1-p} \\
&+\frac{|B||Q|\left\|m_{2}\right\| \psi(r)}{\Gamma(\alpha-\delta+\beta)}\left(\frac{1-p}{\alpha-\delta+\beta-p}\right)^{1-p}\left(1+\eta^{\alpha-\delta+\beta-p}\right), \\
& \sigma=|a|+M\left(\frac{1}{\Gamma(\alpha+1)}+\frac{|Q|\left(1+\eta^{\alpha-1}\right)}{\Gamma(\alpha-\delta+1)}\right), \quad \sup _{t \in[0,1]}|f(t, 0)|=M<\infty .
\end{aligned}
$$


Using $|f(t, x(t))| \leq|f(t, x(t))-f(t, 0)|+|f(t, 0)| \leq L_{1}(t) r+M$ for $x \in B_{r}, t \in[0,1]$, we get

$$
\begin{aligned}
\left|\left(\Phi_{1} x\right)(t)\right| \leq & |A| r\left[\int_{0}^{t} \frac{(t-s)^{\alpha-1}}{\Gamma(\alpha)} L_{1}(s) d s+|Q| \int_{0}^{1} \frac{(1-s)^{\alpha-\delta-1}}{\Gamma(\alpha-\delta)} L_{1}(s) d s\right. \\
& \left.+|Q| \int_{0}^{\eta} \frac{(\eta-s)^{\alpha-\delta-1}}{\Gamma(\alpha-\delta)} L_{1}(s) d s\right]+\sigma|A| \\
\leq & \frac{|A| r}{\Gamma(\alpha)}\left(\int_{0}^{t}(t-s)^{\frac{\alpha-1}{1-p}} d s\right)^{1-p}\left(\int_{0}^{1} L_{1}^{\frac{1}{p}}(s) d s\right)^{p}+\sigma|A| \\
& +\frac{|A||Q| r}{\Gamma(\alpha-\delta)}\left(\int_{0}^{1}(1-s)^{\frac{\alpha-\delta-1}{1-p}} d s\right)^{1-p}\left(\int_{0}^{1} L_{1}^{\frac{1}{p}}(s) d s\right)^{p} \\
& +\frac{|A||Q| r \eta^{\alpha-\delta-p}}{\Gamma(\alpha-\delta)}\left(\int_{0}^{\eta}(\eta-s)^{\frac{\alpha-\delta-1}{1-p}} d s\right)^{1-p}\left(\int_{0}^{1} L_{1}^{\frac{1}{p}}(s) d s\right)^{p} \\
\leq & |A| r\left[\frac{1}{\Gamma(\alpha)}\left(\frac{1-p}{\alpha-p}\right)^{1-p}+\frac{|Q|}{\Gamma(\alpha-\delta)}\left(\frac{1-p}{\alpha-\delta-p}\right)^{1-p}\right. \\
& \left.+\frac{|Q| \eta^{\alpha-\delta-p}}{\Gamma(\alpha-\delta)}\left(\frac{1-p}{\alpha-\delta-p}\right)^{1-p}\right]\left\|L_{1}\right\|+\sigma|A| .
\end{aligned}
$$

In a similar manner, we have that

$$
\begin{aligned}
\left|\left(\Phi_{2} x\right)(t)\right| \leq & |B| \int_{0}^{t} \frac{(t-s)^{\alpha+\beta-1}}{\Gamma(\alpha+\beta)} m(s) \psi(r) d s \\
& +|B||Q| \int_{0}^{1} \frac{(1-s)^{\alpha-\delta+\beta-1}}{\Gamma(\alpha-\delta+\beta)} m(s) \psi(r) d s \\
& +|B||Q| \int_{0}^{\eta} \frac{(\eta-s)^{\alpha-\delta+\beta-1}}{\Gamma(\alpha-\delta+\beta)} m(s) \psi(r) d s \\
\leq & |B| \psi(r)\left[\frac{1}{\Gamma(\alpha+\beta)}\left(\frac{1-p}{\alpha+\beta-p}\right)^{1-p}\right. \\
& \left.+\frac{|Q|}{\Gamma(\alpha-\delta+\beta)}\left(\frac{1-p}{\alpha-\delta+\beta-p}\right)^{1-p}\left(1+\eta^{\alpha-\delta+\beta-p}\right)\right]\|m\| .
\end{aligned}
$$

Thus

$$
\begin{aligned}
|(\Phi x)(t)| \leq & \left|\left(\Phi_{1} x\right)(t)\right|+\left|\left(\Phi_{2} x\right)(t)\right| \\
\leq & |A| r\left[\frac{1}{\Gamma(\alpha)}\left(\frac{1-p}{\alpha-p}\right)^{1-p}+\frac{|Q|}{\Gamma(\alpha-\delta)}\left(\frac{1-p}{\alpha-\delta-p}\right)^{1-p}\right. \\
& \left.+\frac{|Q| \eta^{\alpha-\delta-p}}{\Gamma(\alpha-\delta)}\left(\frac{1-p}{\alpha-\delta-p}\right)^{1-p}\right]\left\|L_{1}\right\|+\sigma|A| \\
& +|B| \psi(r)\left[\frac{1}{\Gamma(\alpha+\beta)}\left(\frac{1-p}{\alpha+\beta-p}\right)^{1-p}\right. \\
& \left.+\frac{|Q|}{\Gamma(\alpha-\delta+\beta)}\left(\frac{1-p}{\alpha-\delta+\beta-p}\right)^{1-p}\left(1+\eta^{\alpha-\delta+\beta-p}\right)\right]\|m\| \\
\leq & \gamma r+\omega \leq r,
\end{aligned}
$$

which implies that $\Phi\left(B_{r}\right) \subset B_{r}$. 
Step 2. $\Phi_{1}$ is continuous and $\gamma$-contractive.

To show the continuity of $\Phi_{1}$ for $t \in[0,1]$, let us consider a sequence $x_{n}$ converging to $x$. Then, by the assumption $\left(\mathrm{H}_{1}\right)$, we have

$$
\begin{aligned}
\left\|\left(\Phi_{1} x_{n}\right)(t)-\left(\Phi_{1} x\right)(t)\right\| \leq & {\left[\frac{|A|\left\|L_{1}\right\|}{\Gamma(\alpha)}\left(\frac{1-p}{\alpha-p}\right)^{1-p}\right.} \\
& \left.+\frac{|A||Q|\left\|L_{1}\right\|}{\Gamma(\alpha-\delta)}\left(\frac{1-p}{\alpha-\delta-p}\right)^{1-p}\left(1+\eta^{\alpha-\delta-p}\right)\right]\left\|x_{n}-x\right\| .
\end{aligned}
$$

Next, we show that $\Phi_{1}$ is $\gamma$-contractive. For $x, y \in B_{r}$, we get

$$
\begin{aligned}
\left\|\left(\Phi_{1} x\right)(t)-\left(\Phi_{1} y\right)(t)\right\| \leq & {\left[\frac{|A|\left\|L_{1}\right\|}{\Gamma(\alpha)}\left(\frac{1-p}{\alpha-p}\right)^{1-p}\right.} \\
& \left.+\frac{|A||Q|\left\|L_{1}\right\|}{\Gamma(\alpha-\delta)}\left(\frac{1-p}{\alpha-\delta-p}\right)^{1-p}\left(1+\eta^{\alpha-\delta-p}\right)\right]\|x-y\| .
\end{aligned}
$$

By the given assumption,

$$
\gamma=\frac{|A|\left\|L_{1}\right\|}{\Gamma(\alpha)}\left(\frac{1-p}{\alpha-p}\right)^{1-p}+\frac{|A||Q|\left\|L_{1}\right\|}{\Gamma(\alpha-\delta)}\left(\frac{1-p}{\alpha-\delta-p}\right)^{1-p}\left(1+\eta^{\alpha-\delta-p}\right)<1,
$$

it follows that $\Phi_{1}$ is $\gamma$-contractive.

Step 3. $\Phi_{2}$ is compact.

In Step 1, it has been shown that $\Phi_{2}$ is uniformly bounded. Now we show that $\Phi_{2}$ maps bounded sets into equicontinuous sets of $C([0,1], \mathbb{R})$. Let $t_{1}, t_{2} \in[0,1]$ with $t_{1}<t_{2}$ and $x \in B_{r}$. Then we obtain

$$
\begin{aligned}
\left\|\left(\Phi_{2} x\right)\left(t_{2}\right)-\left(\Phi_{2} x\right)\left(t_{1}\right)\right\| \leq & \frac{|B|}{\Gamma(\alpha+\beta)} \int_{0}^{t_{1}}\left[\left(t_{2}-s\right)^{\alpha+\beta-1}-\left(t_{1}-s\right)^{\alpha+\beta-1}\right]|g(s, x(s))| d s \\
& +\frac{|B|}{\Gamma(\alpha+\beta)} \int_{t_{1}}^{t_{2}}\left(t_{2}-s\right)^{\alpha+\beta-1}|g(s, x(s))| d s \\
& +|B||Q|\left|t_{2}^{\alpha-1}-t_{1}^{\alpha-1}\right|\left[\int_{0}^{1} \frac{(1-s)^{\alpha-\delta+\beta-1}}{\Gamma(\alpha-\delta+\beta)}|g(s, x(s))| d s\right. \\
& \left.+\int_{0}^{\eta} \frac{(\eta-s)^{\alpha-\delta+\beta-1}}{\Gamma(\alpha-\delta+\beta)}|g(s, x(s))| d s\right] \\
\leq & \frac{|B|}{\Gamma(\alpha+\beta)} \int_{0}^{t_{1}}\left[\left(t_{2}-s\right)^{\alpha+\beta-1}-\left(t_{1}-s\right)^{\alpha+\beta-1}\right] m_{2}(s) d s \\
& +\frac{|B| \psi(r)}{\Gamma(\alpha+\beta)} \int_{t_{1}}^{t_{2}}\left(t_{2}-s\right)^{\alpha+\beta-1} m_{2}(s) d s \\
& +|B||Q| \psi(r)\left|t_{2}^{\alpha-1}-t_{1}^{\alpha-1}\right|\left[\int_{0}^{1} \frac{(1-s)^{\alpha-\delta+\beta-1}}{\Gamma(\alpha-\delta+\beta)} m_{2}(s) d s\right. \\
& \left.+\int_{0}^{\eta} \frac{(\eta-s)^{\alpha-\delta+\beta-1}}{\Gamma(\alpha-\delta+\beta)} m_{2}(s) d s\right] .
\end{aligned}
$$

Obviously, the right-hand side of the above inequality tends to zero independently of $x \in B_{r}$ as $t_{2}-t_{1} \rightarrow 0$. Therefore it follows by the Arzelá-Ascoli theorem that $\Phi_{2}: C([0,1], \mathbb{R}) \rightarrow$ $C([0,1], \mathbb{R})$ is completely continuous. Thus $\Phi_{2}$ is compact on $[0,1]$. 
Step 4. $\Phi$ is condensing.

Since $\Phi_{1}$ is continuous, $\gamma$-contraction and $\Phi_{2}$ is compact, therefore, by Lemma 2.2, $\Phi: B_{r} \rightarrow B_{r}$ with $\Phi=\Phi_{1}+\Phi_{2}$ is a condensing map on $B_{r}$.

Consequently, by Theorem 2.1, the map $\Phi$ has a fixed point which implies that the problem (1) has a solution.

In the special case when $L_{1}(t)=L, L$ a constant, we have the following.

Corollary 3.1 Let $f, g:[0,1] \times \mathbb{R} \rightarrow \mathbb{R}$ be continuous functions. Assume that $g$ satisfies $\left(\mathrm{H}_{2}\right)$ and $f$ satisfies the following condition:

$\left(\mathrm{H}_{1}\right)^{\prime}|f(t, x)-f(t, y)| \leq L|x-y|$ for all $(t, x),(t, y) \in[0,1] \times \mathbb{R}, L>0$ is a constant.

If

$$
\gamma^{\prime}:=\frac{|A| L}{\Gamma(\alpha+1)}+\frac{|A||Q| L}{\Gamma(\alpha-\delta+1)}\left(1+\eta^{\alpha-\delta}\right)<1,
$$

then the boundary value problem (1) has at least one solution.

Example 3.1 Consider a boundary value problem of integro-differential equations of fractional order with nonlocal fractional boundary conditions given by

$$
\left\{\begin{array}{l}
-D^{5 / 2} x(t)=A f(t, x(t))+B I^{\beta} g(t, x(t)), \quad t \in[0,1] \\
D^{1 / 4} x(0)=0, \quad D^{5 / 4} x(0)=0, \quad D^{1 / 4} x(1)-D^{1 / 4} x(\eta)=a
\end{array}\right.
$$

where $A=B=1, \beta=3 / 4, \eta=2 / 3, f(t, x)=\frac{e^{-t}|x|}{\left(1+e^{t}\right)(1+|x|)}, g(t, x)=\frac{t^{2}|x|^{3}}{1+|x|^{3}}$.

Then we have

$$
\begin{aligned}
|f(t, x)-f(t, y)| & =\frac{e^{-t}}{1+e^{t}}\left|\frac{|x|}{1+|x|}-\frac{|y|}{1+|y|}\right| \\
& \leq \frac{e^{-t}|x-y|}{1+e^{t}} \\
& \leq L_{1}(t)|x-y|, \quad \text { where } L_{1}(t)=\frac{1}{2} e^{-t}
\end{aligned}
$$

Also, for all $x \in \mathcal{C}$ and each $t \in[0,1]$, we have

$$
|f(t, x)|=\frac{e^{-t}}{1+e^{t}}\left|\frac{|x|}{1+|x|}\right| \leq \frac{e^{-t}}{1+e^{t}}<m_{1}(t), \quad \text { where } m_{1}(t)=\frac{1}{2} e^{-t}
$$

and $|g(t, x)| \leq m_{2}(t) \psi(\|x\|)$ with $m_{2}(t)=t^{2}\left(\left\|m_{2}\right\|=(3 / 11)^{3 / 4}\right)$ and $\psi(\|x\|)=1$. Selecting $p=3 / 4$ and using the given data, we find that

$$
\begin{aligned}
\gamma & :=\frac{|A|\left\|L_{1}\right\|}{\Gamma(\alpha)}\left(\frac{1-p}{\alpha-p}\right)^{1-p}+\frac{|A||Q|\left\|L_{1}\right\|}{\Gamma(\alpha-\delta)}\left(\frac{1-p}{\alpha-\delta-p}\right)^{1-p}\left(1+\eta^{\alpha-\delta-p}\right) \\
& \simeq 0.825717 .
\end{aligned}
$$

As $\gamma<1$, therefore, by the conclusion of Theorem 3.1, the problem (6) has a solution. 


\section{Competing interests}

The authors declare that they have no competing interests.

\section{Authors' contributions}

Each of the authors, RPA, SKN, BA and MSA, contributed to each part of this study equally and read and approved the final version of the manuscript.

\section{Author details}

'Department of Mathematics, Texas A\&M University, Kingsville, TX 78363-8202, USA. ²Department of Mathematics, Faculty of Science, King Abdulaziz University, P.O. Box 80203, Jeddah, 21589, Saudi Arabia. ${ }^{3}$ Department of Mathematics, University of loannina, loannina, 451 10, Greece.

\section{Acknowledgements}

The authors gratefully acknowledge the referees for their constructive comments that led to the improvement of the original manuscript. This paper was funded by King Abdulaziz University under grant No. (130-1-1433/HiCi). The authors, therefore, acknowledge technical and financial support of KAU.

\section{Received: 15 February 2013 Accepted: 17 April 2013 Published: 6 May 2013}

\section{References}

1. Podlubny, I: Fractional Differential Equations. Academic Press, San Diego (1999)

2. Kilbas, AA, Srivastava, HM, Trujillo, Jj: Theory and Applications of Fractional Differential Equations. North-Holland Mathematics Studies, vol. 204. Elsevier, Amsterdam (2006)

3. Agarwal, RP, Belmekki, M, Benchohra, M: A survey on semilinear differential equations and inclusions involving Riemann-Liouville fractional derivative. Adv. Differ. Equ. 2009, Article ID 981728 (2009)

4. Kirane, M, Malik, SA: The profile of blowing-up solutions to a nonlinear system of fractional differential equations. Nonlinear Anal. 73, 3723-3736 (2010)

5. Kirane, M, Medved, M, Tatar, N-E: Semilinear Volterra integrodifferential problems with fractional derivatives in the nonlinearities. Abstr. Appl. Anal. 2011, Article ID 510314 (2011)

6. Baleanu, D, Mustafa, OG, Agarwal, RP: On $L^{p}$-solutions for a class of sequential fractional differential equations. Appl. Math. Comput. 218, 2074-2081 (2011)

7. Ahmad, B, Nieto, JJ: Riemann-Liouville fractional integro-differential equations with fractional nonlocal integral boundary conditions. Bound. Value Probl. 2011, 36 (2011)

8. Ahmad, B, Ntouyas, SK: A four-point nonlocal integral boundary value problem for fractional differential equations of arbitrary order. Electron. J. Qual. Theory Differ. Equ. 2011, 2 (2011)

9. Sudsutad, W, Tariboon, J: Existence results of fractional integro-differential equations with $m$-point multi-term fractional order integral boundary conditions. Bound. Value Probl. 2012, 94 (2012)

10. Ahmad, B, Ntouyas, SK: Nonlinear fractional differential equations and inclusions of arbitrary order and multi-strip boundary conditions. Electron. J. Differ. Equ. 2012, 98 (2012)

11. Aghajani, A, Jalilian, Y, Trujillo, JJ: On the existence of solutions of fractional integro-differential equations. Fract. Calc. Appl. Anal. 15(2), 44-69 (2012)

12. Carteaa, A, del-Castillo-Negrete, D: Fractional diffusion models of option prices in markets with jumps. Physica A 374 749-763 (2007)

13. Fino, $A Z$, Kirane, $M$ : Qualitative properties of solutions to a time-space fractional evolution equation. Q. Appl. Math. 70, 133-157 (2012)

14. Tao, H, Fu, M, Qian, R: Positive solutions for fractional differential equations from real estate asset securitization via new fixed point theorem. Abstr. Appl. Anal. 2012, Article ID 842358 (2012)

15. Labidi, S, Tatar, N-E: Unboundedness for the Euler-Bernoulli beam equation with fractional boundary dissipation. Appl. Math. Comput. 161, 697-706 (2005)

16. Labidi, S, Tatar, N-E: Breakdown for a Kirchhoff type beam with a fractional boundary feedback. J. Dyn. Control Syst. $14,71-94(2008)$

17. Granas, A, Dugundji, J: Fixed Point Theory. Springer, New York (2003)

18. Zeidler, E: Nonlinear Functional Analysis and Its Application: Fixed Point-Theorems, vol. 1. Springer, New York (1986)

19. Sadovskii, BN: On a fixed point principle. Funct. Anal. Appl. 1, 74-76 (1967)

doi:10.1186/1687-1847-2013-128

Cite this article as: Agarwal et al.: Existence of solutions for integro-differential equations of fractional order with nonlocal three-point fractional boundary conditions. Advances in Difference Equations 2013 2013:128. 